\title{
Performance of Series Connected GaAs Photovoltaic Converters under Multimode Optical Fiber Illumination
}

\author{
Tiqiang Shan and Xinglin Qi \\ The Third Department, Mechanical Engineering College, Shijiazhuang 050000, China \\ Correspondence should be addressed to Tiqiang Shan; stq0701@163.com
}

Received 26 September 2014; Accepted 6 December 2014; Published 21 December 2014

Academic Editor: Armin Gerhard Aberle

Copyright (c) 2014 T. Shan and X. Qi. This is an open access article distributed under the Creative Commons Attribution License, which permits unrestricted use, distribution, and reproduction in any medium, provided the original work is properly cited.

\begin{abstract}
In many military and industrial applications, GaAs photovoltaic (PV) converters are connected in series in order to generate the required voltage compatible with most common electronics. Multimode optical fibers are usually used to carry high-intensity laser and illuminate the series connected GaAs PV converters in real time. However, multimode optical fiber illumination has a speckled intensity pattern. The series connected PV array is extremely sensitive to nonuniform illumination; its performance is limited severely by the converter that is illuminated the least. This paper quantifies the effects of multimode optical fiber illumination on the performance of series connected GaAs PV converters, analyzes the loss mechanisms due to speckles, and discusses the maximum illumination efficiency. In order to describe the illumination dependent behavior detailedly, modeling of the series connected PV array is accomplished based on the equivalent circuit for PV cells. Finally, a series of experiments are carried out to demonstrate the theory analysis.
\end{abstract}

\section{Introduction}

An increasing application of GaAs PV converters is the conversion of monochromatic light into electrical power [15]. A typical application of this technology is the optical transmission of energy or power-by-light. Electrical energy delivery in power-by-light systems represents a promising alternative for copper wires [6]. In such systems, the laser light is mostly transmitted through an optical fiber and a PV receiver converts the optical energy into electrical energy. These systems are used in places where the use of electrical energy is not recommendable, taking advantage of the properties of optical fiber such as electrical insulation, immunity from EMI, RF, and lightning. Examples of these applications are found in fields such as the oil industry, remote sensing, aerospace, explosions, high voltage power lines, and nuclear plants $[7,8]$. In these applications, the required voltage of the electronic circuit is generally higher than that supplied by a single GaAs PV converter; this value is about $1 \mathrm{~V}$. Therefore, GaAs converters are always connected in series to match the required voltage. One of the principal advantages to pursuing this technology is that electric power can be generated in a far smaller volume than required by a DC-DC converter. This option is more compact and has the possibility of integrating the GaAs PV converters with an electronic circuit.

Fiber illumination is important because some of today's optical power transfer applications do not have line of sight between the optical source and series connected PV converters. The optical power is delivered via optical fiber to a region with insufficient volume to afford beam-shaping optics with which to uniformly illuminate the series connected PV converters. It has been shown that the least illuminated converter dominates the system performance [9]. It is this dilemma that leads to the need for research into the performance of series connected GaAs PV converters under fiber coupled laser diode illumination.

Large core, step-index multimode optical fibers are usually used to carry high power levels over short distances. Multimode optical fiber illumination has a speckled intensity pattern and the illumination efficiency is a function of the ratio of the individual converter area within the series connected PV arrays to the speckle correlation area. Nonuniform 


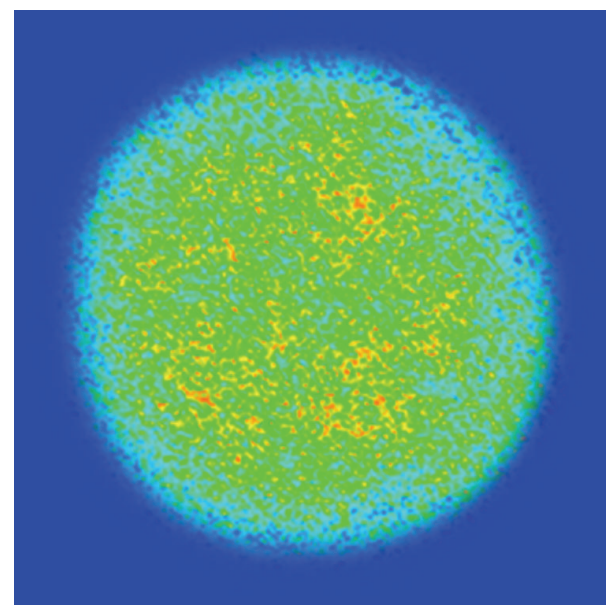

FIGURE 1: The illumination profile of step-index multimode fiber.

illumination could reduce the efficiency of electric conversion and decrease the lifetime of PV converters [10]. This paper quantifies the effects of step-index multimode optical fiber nonuniform illumination on the performance of series connected GaAs PV converters, analyzes the loss mechanisms due to speckles, and discusses the maximum illumination efficiency under multimode illumination. In order to describe the illumination dependent behavior detailedly, an analytical model of the series connected PV converters is accomplished based on the equivalent circuit for PV cells. To demonstrate the theory analysis above, finally, a series of experiments are carried out.

\section{Multimode Optical Fiber Illumination}

The operating voltage of the series connected PV converters in the string is the sum of the operating voltages of the subconverters, whereas the current through each subconverter is the same and equal to the current of the external circuit. The converter generating the lowest short circuit current, referred to as the individual mismatched converter, determines the system current and the device performance [11]. Therefore, in the case of series connected GaAs PV converters, nonuniform illumination can cause a significant efficiency drop. This makes the spatial profile of the illumination source a critical parameter.

2.1. Mean Speckle Correlation Area. The illumination profile produced under step-index multimode optical fiber illumination contains speckles due to the interference of the different modes. Speckles are random regions of fluctuation in the intensity profile [12]. If the speckle is large enough it can create a situation where converters within a series connected GaAs PV array are weakly illuminated, thereby limiting the current. The illumination profile from a stepindex multimode fiber shown in Figure 1 is speckled; the illumination efficiency is a function of the ratio of the individual converter area to the speckle correlation area.
This section is based on a detailed discussion of the statistical properties of speckle [13]. The mean speckle correlation area is calculated by evaluating the normalized mutual intensity of the speckled intensity pattern incident upon the series connected PV array [13, 14]. The mean speckle correlation area of an intensity pattern propagating from a step-index multimode optical fiber is given by

$$
S_{C}=\frac{\lambda^{2} z^{2}}{\pi a^{2}}
$$

where $a$ is the core diameter of the fiber, $\lambda$ is the wavelength, and $z$ is the propagation distance from the fiber.

The speckle correlation area is directly related to both the propagation distance and wavelength and inversely related to the fiber core diameter. Keeping the fiber core diameter to a maximum while minimizing the wavelength and propagation distance is critical in reducing the size of the speckle and therefore increasing the illumination efficiency.

2.2. Illumination Efficiency. The amplitude of the generated photocurrent, $I_{P}$, is determined by

$$
I_{P}=I_{L} S_{D} R
$$

where $I_{L}$ is the irradiance $\left(\mathrm{W} / \mathrm{cm}^{2}\right), S_{D}$ is the area of a single converter $\left(\mathrm{cm}^{2}\right)$, and $R$ is the wavelength dependent responsivity (A/W) [15].

Speckle is a random phenomenon and as such must be treated statistically. The illumination efficiency, $E_{I}$, is determined by the converter that is illuminated the least. To begin this discussion, we define $E_{I}$ for multimode optical fiber illumination as

$$
\begin{aligned}
E_{I} & =\frac{I_{S \min } S_{D} R}{\left\langle I_{S}\right\rangle S_{D} R}=\frac{I_{S \min }}{\left\langle I_{S}\right\rangle}=\frac{\left\langle I_{S}\right\rangle-\sigma_{I}}{\left\langle I_{S}\right\rangle} \\
& =1-\frac{\sigma_{I}}{\left\langle I_{S}\right\rangle}=1-\frac{1}{\sqrt{\mu}},
\end{aligned}
$$

where $\left\langle I_{S}\right\rangle$ is the mean integrated speckled intensity across any individual converter within the array, $I_{S \min }$ is the minimum integrated speckled intensity across an individual converter within the array, and $\sigma_{I}$ is the standard deviation of the integrated speckle pattern [14].

The variable $\mu$ is a function of the ratio of the converter area, $S_{D}$, to the speckle correlation area, $S_{C}$, and is defined as

$$
\frac{\left\langle I_{S}\right\rangle}{\sigma_{I}}=\sqrt{\mu}
$$

An exact expression for $\mu$ is found for a square aperture which is analogous to an individual square PV cell [14],

$$
\mu=\left[\sqrt{\frac{S_{C}}{S_{D}}} \operatorname{erf}\left(\pi \frac{S_{D}}{S_{C}}\right)-\left(\frac{S_{C}}{\pi S_{D}}\right)\left(1-\exp \left(-\pi \frac{S_{D}}{S_{C}}\right)\right)\right]^{-2} .
$$




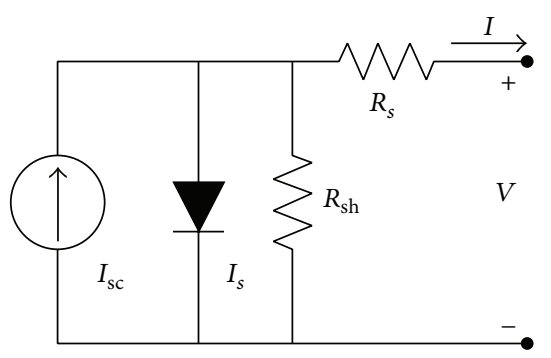

(a)

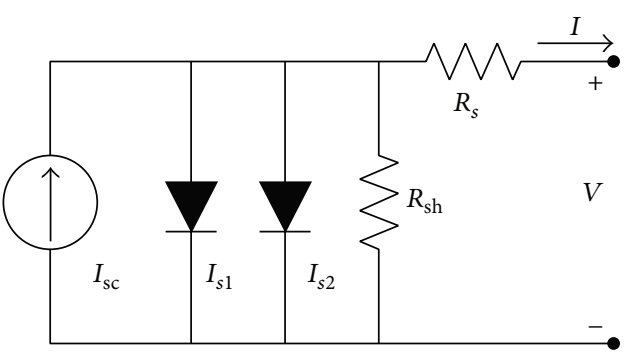

(b)

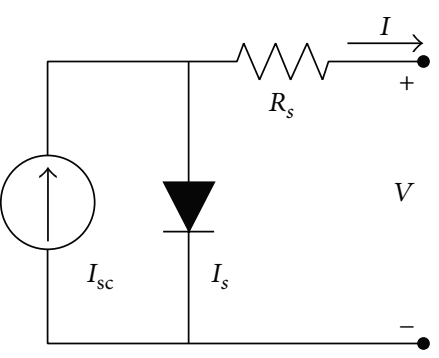

(c)

FIGURE 2: PV cells equivalent circuit models, (a) single-diode model, (b) double-diode model, and (c) simplified model.

This equation is then modified for a rectangular PV cell and is now in the form of

$$
\begin{aligned}
\mu=\{[ & \sqrt{\frac{S_{C}}{L_{1}^{2}}} \operatorname{erf}\left(\pi \frac{L_{1}^{2}}{S_{C}}\right)-\left(\frac{S_{C}}{\pi L_{1}^{2}}\right) \\
& \left.\times\left(1-\exp \left(-\pi \frac{L_{1}^{2}}{S_{C}}\right)\right)\right] \\
& \times\left[\sqrt{\frac{S_{C}}{L_{2}^{2}}} \operatorname{erf}\left(\pi \frac{L_{2}^{2}}{S_{C}}\right)-\left(\frac{S_{C}}{\pi L_{2}^{2}}\right)\right. \\
& \left.\left.\times\left(1-\exp \left(-\pi \frac{L_{2}^{2}}{S_{C}}\right)\right)\right]\right\}^{-1},
\end{aligned}
$$

where $L_{1}$ and $L_{2}$ are the dimensions of the rectangular cell.

The minimum integrated speckled intensity is defined as being one standard deviation below the mean in (3). As such, it is an upper limit for the illumination efficiency. The actual value may be lower due to the probability of having a value of $I_{S \min }$ that is lower than one standard deviation below the mean. In addition, nonuniform intensity envelope across the series connected PV converters will also lower the illumination efficiency.

\section{Mathematical Model}

Modeling of the series connected PV converters is accomplished based on the equivalent circuit for PV cells. The traditional models of PV cells represented by a current source in parallel with one or two diodes are shown in Figure 2. The single-diode model shown in Figure 2(a) includes four components: photocurrent source, diode parallel to the source, series resistor $R_{s}$, and shunt resistor $R_{\mathrm{sh}}$. In double-diode model shown in Figure 2(b), an extra diode is added for better curve fitting in low irradiance level conditions [16]. In most cases, due to the exponential equation of a $p-n$ diode junction, it is difficult to determine these five parameters in single-diode model or six parameters in double-diode model mathematically. Since the shunt resistance $R_{\mathrm{sh}}$ is large, it can usually be neglected [17]. The single-diode model and doublediode model can, thus, be simplified into Figure 2(c), an equivalent circuit model of this study.
The light-generated current of the PV cell depends linearly on the illumination. At a certain optical power $P_{\text {in }}$ with the wavelength $\lambda$, the short circuit current $I_{\mathrm{sc}}$ for an ideal $\mathrm{PV}$ cell is given by

$$
I_{\mathrm{sc}}=\frac{q \lambda}{h c} P_{\text {in }} Q_{\text {ext }}(\lambda),
$$

where $h$ is the Planck constant, $c$ is the velocity of light in vacuum, $q$ is the electronic charge, and $Q_{\text {ext }}(\lambda)$ is the external quantum efficiency (QE).

According to the simplified model of PV cells, the illuminated current $I$ and voltage $V$ are represented as

$$
I=I_{\mathrm{sc}}-I_{s 0}\left(\exp \left(\frac{V+I R_{s}}{V_{t}}\right)-1\right)
$$

where $V_{t}=n k T / q$, on the assumption that $T=300 \mathrm{~K}$ and $n=$ $1, V_{t} \approx 25 \mathrm{mV}$, and $I_{s 0}, R_{s}$, and $n$ are the saturation current, series resistance, and ideality factor, respectively.

In respect that $\exp \left(\left(V+I R_{s}\right) / V_{t}\right) \gg 1$ [18], the illuminated current $I$ and voltage $V$ can be represented as

$$
I=I_{\mathrm{sc}}-I_{s 0} \exp \left(\frac{V+I R_{s}}{V_{t}}\right) .
$$

At the open circuit state, the saturation current $I_{s 0}$ is obtained as

$$
I_{s 0}=I_{\mathrm{sc}} \exp \left(-\frac{V_{\mathrm{oc}}}{V_{t}}\right)
$$

The output electrical power $P_{\text {out }}$ of PV cells is given by $P=I V$. In principle, the current $I_{m}$ and the voltage $V_{m}$ at the maximum output power can be obtained from the condition of $d P / d V=0$, but the educed expressions are of great complexity. According to Antonio and Steven [18], one empirical expression can be used to represent this relation:

$$
F F=\frac{I_{m} V_{m}}{I_{\mathrm{sc}} V_{\mathrm{oc}}}=F F_{0}\left(1-r_{s}\right),
$$

where $F F_{0}=\left(v_{\mathrm{oc}}-\ln \left(v_{\mathrm{oc}}+0.72\right)\right) /\left(v_{\mathrm{oc}}+1\right), v_{\mathrm{oc}}=V_{\mathrm{oc}} / V_{t}$, and $r_{s}=R_{s} I_{\mathrm{sc}} / V_{\mathrm{oc}}$.

Using (11), the series resistance is given by

$$
R_{s}=\left(1-\frac{F F}{F F_{0}}\right) \frac{V_{\mathrm{oc}}}{I_{\mathrm{sc}}}
$$


All expressions mentioned above would be effectual, on condition that $v_{\mathrm{oc}}>15$ and $r_{s}<0.4$. In general, the precision is higher than $1 \%$ [18]. For GaAs PV cells, it is reasonable to assume that $V_{\mathrm{oc}}=1.0 \mathrm{~V}$; thus, $v_{\mathrm{oc}}=40>15, F F_{0}=0.89$. Another condition is that $r_{s}<0.4$; namely, require $F F>$ $0.6 F F_{0}=0.53$; in fact, this condition can be reached easily for GaAs PV cells.

When $N$ identical PV converters are connected in series and placed under nonuniform illumination, the $I-V$ curve of series connected PV converters is best expressed in the form of a summation of logarithmic terms,

$$
\begin{aligned}
& V(I)=\sum_{i=1}^{N}\left[V_{t} \ln \left(\frac{I_{\mathrm{sci}}-I}{I_{s 0}}\right)-I R_{s i}\right], \\
& V(I)=\sum_{i=1}^{N}\left[V_{t} \ln \left(\frac{I_{\mathrm{sci}}-I}{I_{s 0}}\right)-I\left(1-\frac{F F}{F F_{0}}\right) \frac{V_{\mathrm{oc}}}{I_{\mathrm{sci}}}\right],
\end{aligned}
$$

where $I$ is limited by the illumination current generated from the least illuminated converter $\left(I_{\mathrm{sci}}\right)$.

\section{Experiments and Analysis}

The current limiting effect of the least illuminated converter is illustrated with an experiment where an illuminated series connected GaAs PV array was partially shadowed. The series connected GaAs PV array consists of 6 converters. In this experiment, a $105 \mu \mathrm{m}$ core diameter, $0.22 \mathrm{NA}$ step-index multimode optical fiber is adopted. PV converters based on GaAs are mostly used for the conversion of red or nearinfrared laser light. In our experiments, the illumination source is an $808 \mathrm{~nm}$ multimode laser with a maximum output power of $94 \mathrm{WCW}$, and the spectral width of the laser source is about $4 \mathrm{~nm}$. An entire row consisting of three series connected cells was shadowed incrementally and the short circuit current was recorded. As shown in Figure 3, there is a linear relationship between the percent shadow and percent decrease in output current. The current is greatly reduced when the entire row is shadowed. Diffraction and reflection account for the less than $100 \%$ decrease in output current when the entire row is completely shadowed.

Illumination efficiency when using multimode illumination is dependent on the ratio of the PV converter area to the mean speckle correlation area. This theory is demonstrated by measuring the output current relative to the optical power incident upon the series connected PV converters at the distances of which the speckle correlation area and power were determined. To achieve a wider ratio of PV converter area to mean speckle correlation area, the mean speckle correlation area is increased by increasing the distance between the series connected PV converters and the fiber. The decreased illumination intensity at the greater distances was compensated for in the experimental calculations. The illumination efficiency is calculated by

$$
E_{I}=\frac{I_{\text {out }}}{P_{u} R},
$$

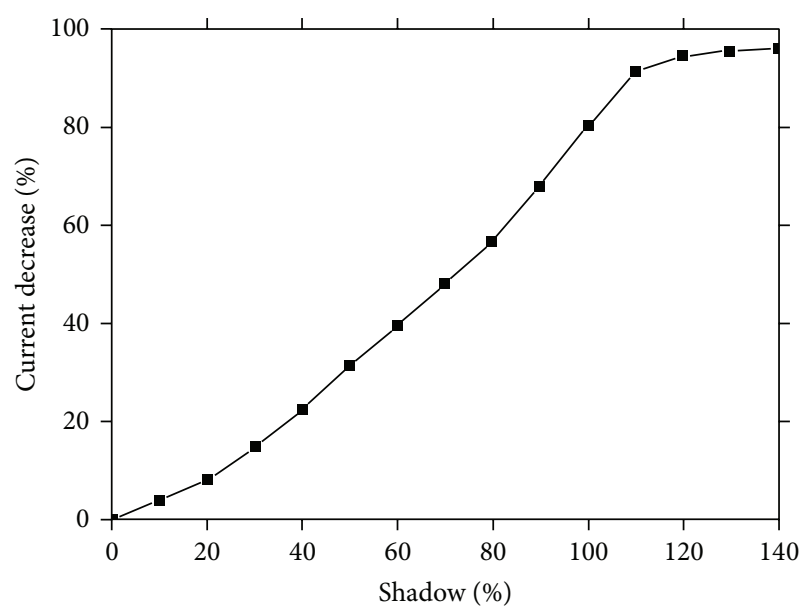

FIGURE 3: The current limiting effect of the least illuminated converter.

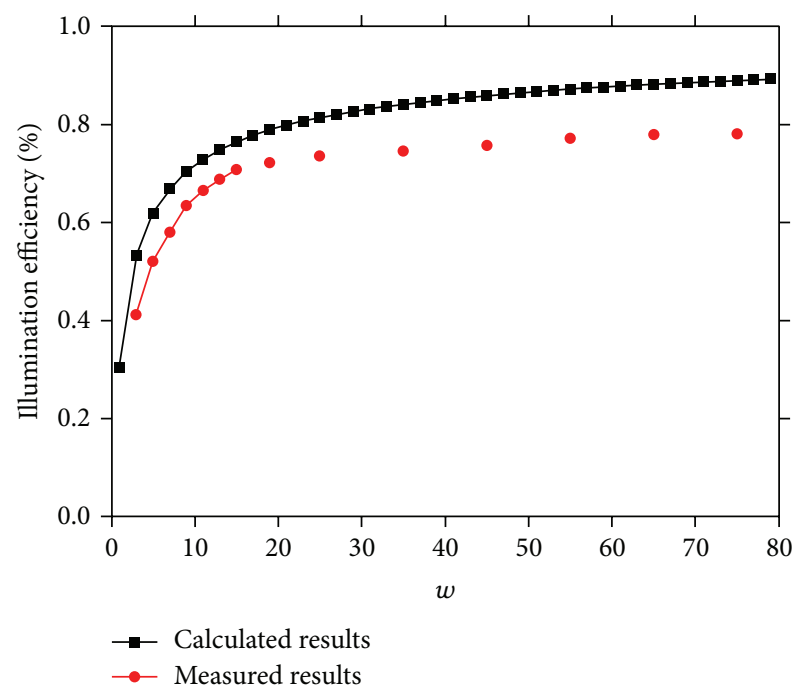

FIGURE 4: The illumination efficiency as a function of PV converter area to mean speckle correlation area.

where $E_{I}$ is the ratio of the measured output current, $I_{\text {out }}$, to the current that would be produced given a uniform beam, $P_{u}$.

Figure 4 shows the experimental results with the illumination efficiency plotted as a function of the ratio of PV converter area to mean speckle correlation area, $w$. The measured results are found to be in close agreement with the theoretical prediction in trend, but the measured efficiencies are lower than predicted. This can be explained by the way that the illumination efficiency is defined. As mentioned earlier, the illumination efficiency is defined as the minimally illuminated converter in the series connected PV array being illuminated at a level that is a standard deviation below the mean. Probabilities exist that the minimally illuminated converter will be illuminated at a level lower than a standard deviation below the mean. In addition, the intensity envelope 


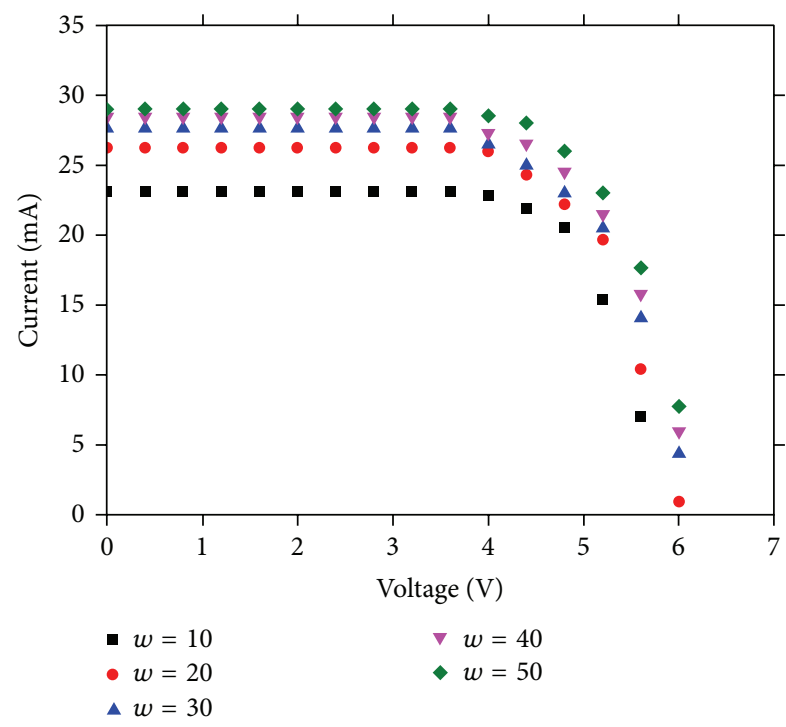

FIGURE 5: $I-V$ characteristics for different ratios of PV converter to mean speckle correlation area.

was nonuniform which also contributes to a decrease in illumination efficiency.

Experimental determination of the $I-V$ characteristics of series connected GaAs PV converters is required for its application. The efficiency can be calculated from the maximum power point of the measured $I-V$ curve and the determined illumination efficiency. The optical loss that results from shadowing by the metal lines and from sacrifice of active light receiving area for formation of the isolation channels was compensated for in the experimental calculations. In previous experiments, the conversion efficiency of $51.9 \%$ at $1 \mathrm{~W} / \mathrm{cm}^{2}$ has been achieved for a single GaAs PV converter with $I_{\mathrm{sc}}=36.38 \mathrm{~mA}, V_{\mathrm{oc}}=1.07 \mathrm{~V}$, and $P_{\max }=31.14 \mathrm{~mW}$. Figure 5 shows the $I-V$ characteristics of series connected $\mathrm{PV}$ converters measured at $1 \mathrm{~W} / \mathrm{cm}^{2}$, for different values of $w(10,20,30,40$, and 50$)$. The measured data can serve to the influence of nonuniform illumination that worsen the device performance.

Also presented in experiment results are properties of the short circuit current, open circuit voltage, maximum output power, and conversion efficiency of the series connected GaAs PV converters. As seen in Table 1, the open circuit voltage increases slightly with $w$ increasing; an important factor penalizing the efficiency is the decrease in the short circuit current that results from the speckle under multimode optical fiber illumination.

\section{Conclusions}

Multimode optical fiber illumination has a speckled intensity pattern. Therefore, the main subject of this paper is to evaluate the effects of step-index multimode optical fiber nonuniform illumination on the performance of series connected GaAs PV converters. The current limiting effect of the least illuminated converter is illustrated with an experiment. As
TABLE 1: Measured results of the performance parameters.

\begin{tabular}{lcccc}
\hline$w$ & $I_{\text {sc }}(\mathrm{mA})$ & $V_{\mathrm{oc}}(\mathrm{V})$ & $P_{\max }(\mathrm{mW})$ & $\eta(\%)$ \\
\hline 10 & 23.08 & 5.93 & 109.5 & 30.4 \\
20 & 26.24 & 6.04 & 126.8 & 35.2 \\
30 & 27.61 & 6.18 & 136.5 & 37.9 \\
40 & 28.44 & 6.23 & 142.0 & 39.4 \\
50 & 29.01 & 6.30 & 146.4 & 40.7 \\
\hline
\end{tabular}

shown in experiment results, there is a linear relationship between the percent shadow and percent decrease in output current of the series connected PV converters under multimode illumination. Illumination efficiency is determined by the ratio of the converter area to the speckle correlation area. Theoretical study demonstrates that keeping the fiber diameter to a maximum while minimizing the wavelength and propagation distance is critical in reducing the size of the speckle and thus increasing the illumination efficiency. Although the measured data of the illumination efficiency is lower than predicted due to the probabilities that the minimally illuminated converter will be illuminated at a level lower than a standard deviation below the mean, the measured results are found to be in close agreement with the theoretical prediction in trend. The data in both cases shows that illumination efficiency decreases rapidly for ratios less than 10. In addition, the $I-V$ characteristics of series connected PV converters measured at $1 \mathrm{~W} / \mathrm{cm}^{2}$, for different converter/speckle ratios, serve to the influence of the speckles that worsen the device performance.

\section{Conflict of Interests}

The authors declare that there is no conflict of interests regarding the publication of this paper.

\section{References}

[1] D. Krut, R. Sudharsanan, W. Nishikawa, T. Isshiki, J. Ermer, and N. H. Karam, "Monolithic multi-cell GaAs laser power converter with very high current density," in Proceedings of the 29th IEEE Photovoltaic Specialists Conference, pp. 908-911, New Orleans, La, USA, May 2002.

[2] R. Peña, C. Algora, and I. Anton, "GaAs multiple photovoltaic converters with an efficiency of $45 \%$ for monochromatic illumination," in Proceddings of the 3rd World Conference on Photovoltaic Energy Conversion, pp. 228-231, Osaka, Japan, May 2003.

[3] G. Böttger, M. Dreschmann, C. Klamouris et al., "Optically powered video camera link," in Proceedings of the 33rd European Conference and Exhibition on Optical Communication (ECOC '07), pp. 123-124, Berlin, Germany, September 2007.

[4] S. J. Wojtczuk, "Long-wavelength laser power converters for optical fibers," in Proceedings of the IEEE 26th Photovoltaic Specialists Conference, pp. 971-974, Anaheim, Calif, USA, October 1997.

[5] E. Oliva, F. Dimroth, and A. W. Bett, "GaAs converters for high power densities of laser illumination," Progress in Photovoltaics: Research and Applications, vol. 16, no. 4, pp. 289-295, 2008. 
[6] R. Peña and C. Algora, "Assessment of photovoltaic converters and other components for power-by-light systems," in Proceedings of the 20th European Conference on Photovoltaic Solar Energy, pp. 488-491, Barcelona, Spain, 2005.

[7] J. Schubert, E. Oliva, F. Dimroth, W. Guter, R. Loeckenhoff, and A. W. Bett, "High-voltage GaAs photovoltaic laser power converters," IEEE Transactions on Electron Devices, vol. 56, no. 2, pp. 170-175, 2009.

[8] R. Peña and C. Algora, "Evaluation of mismatch and nonuniform illumination losses in monolithically series-connected GaAs photovoltaic converters," Progress in Photovoltaics: Research and Applications, vol. 11, no. 2, pp. 139-150, 2003.

[9] H. Rauschenbach, Solar Cell Array Design Handbook, Van Nostrand Reinhold, New York, NY, USA, 1980.

[10] H. Baig, K. C. Heasman, and T. K. Mallick, "Non-uniform illumination in concentrating solar cells," Renewable and Sustainable Energy Reviews, vol. 16, no. 8, pp. 5890-5909, 2012.

[11] R. Peña and C. Algora, "Mismatch losses in GaAs monolithically series connected photovoltaic converters for monochromatic illumination," in Proceedings of the 16th European Conference on Photovoltaic Solar Energy, pp. 1038-1041, Glasgow, Scotland, 2000.

[12] J. W. Shelton, F. M. Dickey, and S. Krishna, "Series connected photovoltaic array performance under non-uniform illumination," in Optical Technologies for Arming, Safing, Fuzing, and Firing IV, 70700R, vol. 7070 of Proceedings of SPIE, pp. 1-8, San Diego, Calif, USA, August 2008.

[13] J. Goodman, Statistical Optics, John Wiley \& Sons, New York, NY, USA, 1985.

[14] J. Goodman, Statistical Properties of Laser Patterns, Springer, Berlin, Germany, 1984.

[15] E. Dereniak and D. Crowe, Optical Radiation Detectors, John Wiley \& Sons, New York, NY, USA, 1984.

[16] Z. Salam, K. Ishaque, and H. Taheri, "An improved two-diode photovoltaic (PV) model for PV system," in Proceedings of the Joint International Conference on Power Electronics, Drives and Energy Systems (PEDES '10), pp. 131-137, New Delhi, India, December 2010.

[17] X. Weidong, W. G. Dunford, and A. Capel, "A novel modeling method for photovoltaic cells," in Proceedings of the IEEE 35th Annual Power Electronics Specialists Conference (PESC '04), pp. 1950-1956, Anaheim, Calif, USA, June 2004.

[18] L. Antonio and H. Steven, Handbook of Photovoltaic Science and Engineering, John Wiley \& Sons, New York, NY, USA, 2003. 

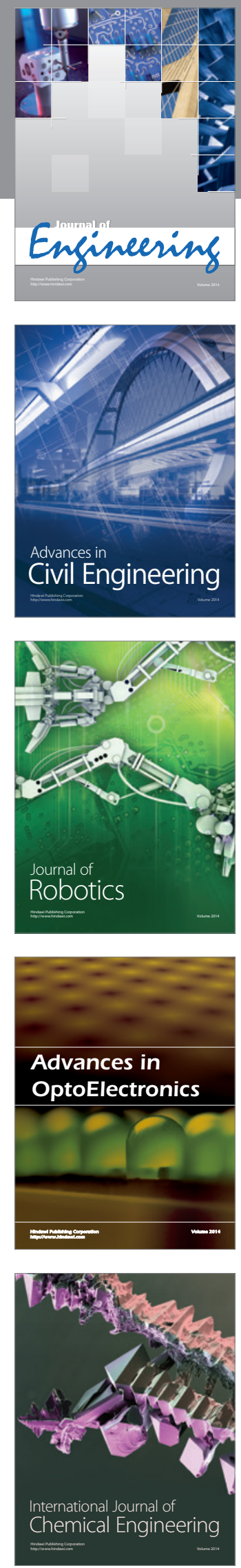

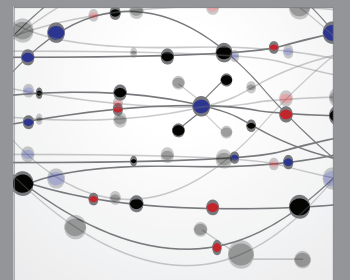

The Scientific World Journal
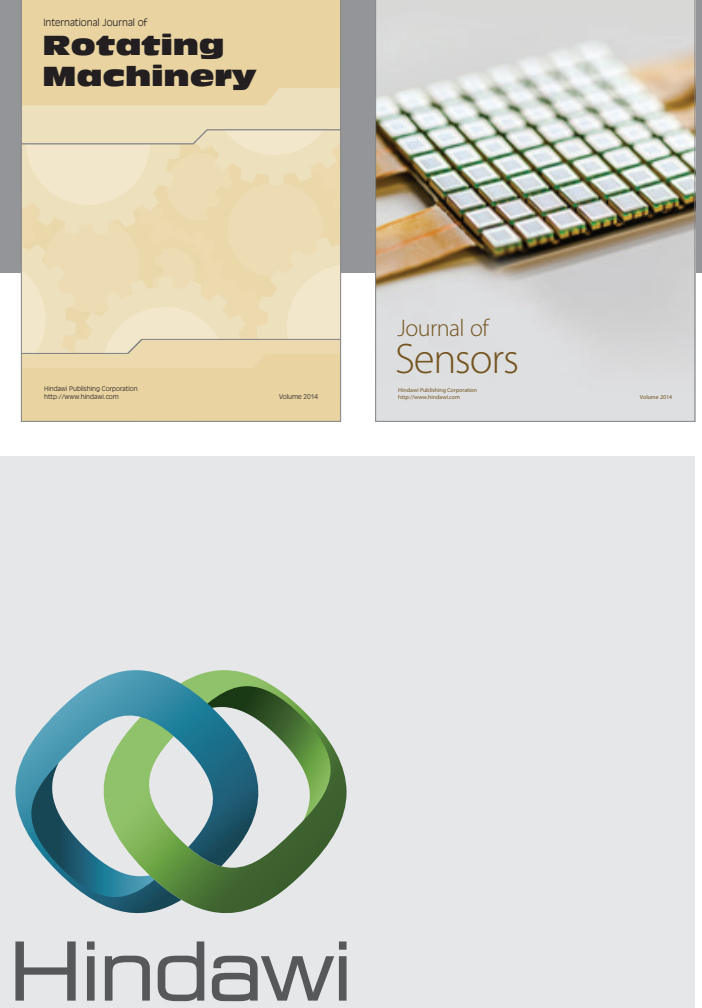

Submit your manuscripts at http://www.hindawi.com
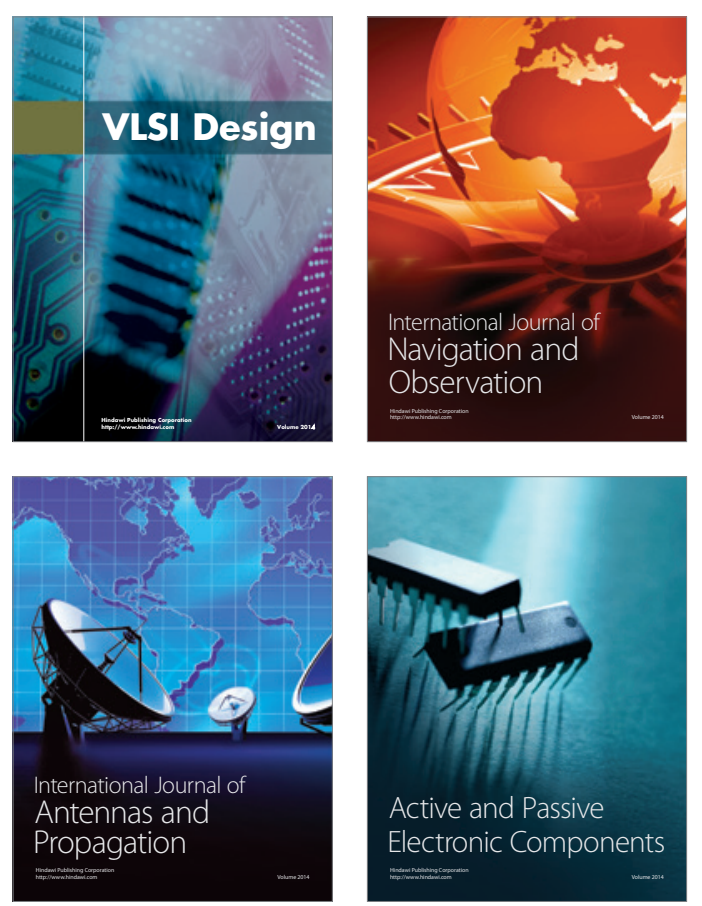
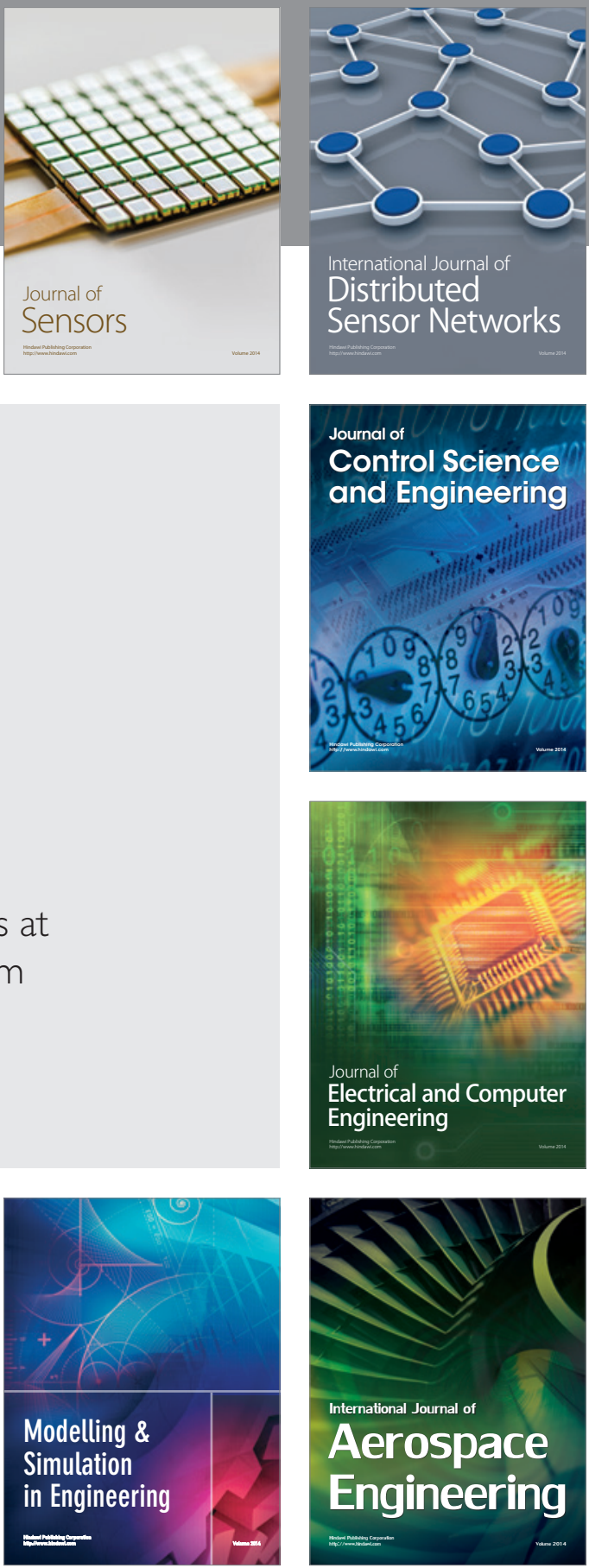

Journal of

Control Science

and Engineering
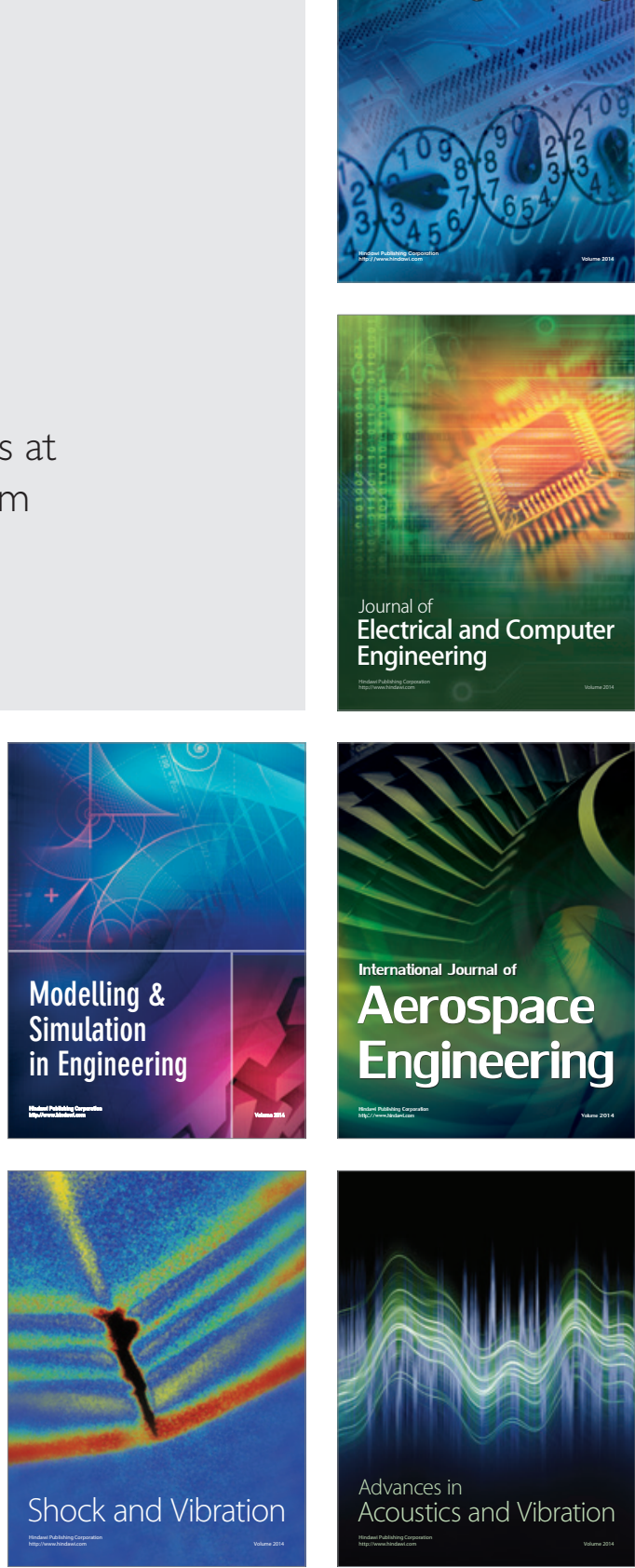